Estudios Románicos, Volumen 27, 2018, pp. 253-264

ISSN: 0210-491

eISSN: $1989-614 \mathrm{X}$

DOI: https://doi.org/10.6018/ER/346681

\title{
DIALEGESTHAI. IL DIALOGO COME PRATICA IN MICHELSTAEDTER
}

(Dialegesthai. Dialogue as a Practice in Michelstaedter)

\author{
Claudia Zavaglini* \\ Univerzita Palackého v Olomouci
}

\begin{abstract}
This contribution focuses on the role and importance of dialogue in Michelstaedter's life and works. In the first part, I demonstrate how his writing displays a certain dialogic intentionality, expressed through the omnipresent second person singular, dialogism - in support of his arguments - and also "pure" dialogue. In the last part, I investigate the reasons behind these choices, suggesting a correlation between form and the larger purpose of his writing.
\end{abstract}

Key-words: Michelstaedter; Dialogue; Dialogism; Dialectics; Mimesis.

Riassunto: Il contributo si sofferma sulle declinazioni della dialogicità in Michelstaedter e ne descrive l'importanza a livello sia biografico che testuale, evidenziando come la pratica del dialogo si profili in tutti e due i casi come scelta consapevole. La scrittura risulta contaminata dall'intenzionalità dialogica, che si esprime talvolta nell'onnipresenza del $t u$ nel testo, talvolta nell'utilizzo del dialogismo a supporto dell'argomentazione, fino all'uso del dialogo puro. Nella parte conclusiva vengono ipotizzate le motivazioni della scelta dialogica e, attraverso Il dialogo della salute, la correlazione tra forma e finalità della scrittura.

Parole chiave: Michelstaedter; Dialogo; Dialogismo; Dialettica; Mimesis.

Io ho piacere a star con lui, e credo che non sia male d'aver qualcuno che ci ricordi che non siam vissuti e non viviamo come si dovrebbe.

Platone, Lachete

La potenza della parola è solo della dialettica.

Michelstaedter, Appendici critiche

* Indirizzo per la corrispondenza: Claudia Zavaglini, Katedra romanistiky. Filozofická fakulta Univerzity Palackého v Olomouci, Křížkovského 10 - 77900 Olomouc, Česká republika [claudia.zavaglini01@upol.cz]. 


\section{Introduzione ${ }^{1}$}

Carlo Michelstaedter è conosciuto e studiato in primis per quella che viene considerata la sua opera maggiore, La persuasione e la rettorica, o meglio per il suo primo nucleo, poiché le Appendici critiche, che ne sono parte integrante, hanno avuto fortuna e diffusione ben diverse. Tra gli scritti meno letti, oltre alle Appendici, si possono annoverare senz'altro i dialoghi, almeno se si fa eccezione per Il dialogo della salute, l'esempio più corposo e vivo di quella dialogicità che contraddistingue e permea la scrittura di Michelstaedter. Il dialogo della salute, insieme alla Poesie, è la prima opera di Michelstaedter ad essere pubblicata (Michelstaedter 1912).

Nonostante la volontà del curatore (e intimo amico di Michelstaedter) Vladimiro Arangio Ruiz di avviare la pubblicazione degli scritti partendo da La persuasione e la rettorica, ad avere l'ultima parola è l'editore Formiggini, che in una lettera del 27 settembre 1911 precisa: «io debbo pensare alla opportunità editoriale: le poesie interessano un pubblico molto più ampio, insisto perciò vivamente sulla opportunità di pubblicare prima le poesie e il dialogo in un volume» (Meroi 2011: 139); così, le prime opere ad essere stampate, per «opportunità editoriale», sono appunto Il dialogo della salute e una selezione delle poesie. In seguito, il Dialogo è stato ristampato nel 1958, nel volume Sansoni delle Opere curato da Chiavacci. In questa edizione, tra gli Scritti vari, vengono pubblicati, non sempre integralmente, anche altri dialoghetti: il dialogo con Nadia, il dialogo tra Diogene e Napoleone, l'inizio di un dialogo sul sentimento, il dialogo tra la cometa e la Terra, il dialogo tra l'adolescente e l'uomo, il dialogo tra il borghese e il saggio, il dialogo tra la folla e l'uomo, il dialogo tra Michelstaedter e Socrate e anche la prima versione del finale del Dialogo della salute. Un altro dialogo, che ha come protagonista il personaggio principale del romanzo Il fuoco di D'Annunzio, Stelio Effrena, viene pubblicato da Cerruti tra i testi inediti che accompagnano il suo saggio (Cerruti 1987: 243-247).

Il merito di aver raccolto in un unico volume Il dialogo della salute e la maggior parte di questi dialoghetti va al curatore delle edizioni Adelphi delle opere di Michelstaedter, Sergio Campailla. Dopo La persuasione e la rettorica (1982), l'Epistolario (1983) e le Poesie (1987), nel 1988 esce nella Piccola Biblioteca Adelphi il volumetto Il dialogo della salute e altri dialoghi, nel quale vengono riuniti e ristampati quasi tutti i dialoghi già editi da Chiavacci. Il dialogo della salute è stato edito nello stesso anno anche da Agalev (Michelstaedter 1988b) e più recentemente, nel 2010, da Mimesis (Michelstaedter 2010).

Ad oggi, lo studio più puntuale sulla rilevanza della componente dialogica in Michelstaedter è quello di Roberta Tanzi (2003), che ha il merito di sottolineare la complementarietà del Dialogo della salute rispetto a La persuasione e la rettorica e la peculiarità della scelta dialogica. L'uso del dialogo non è in Michelstaedter una scelta indifferente o casuale, ma un elemento che conferma ulteriormente la profonda unità e l'inscindibilità di vita e pensiero (e dunque scrittura), tanto spesso sottolineata in sede

1 Il presente studio è stato finanziato dai progetti IGA_FF_2015_027 e IGA_FF_2017_043 del Dipartimento di Lingue romanze dell’Università Palacký di Olomouc. 
critica. Premesso tutto questo, il presente contributo intende soffermarsi sulla componente dialogica, al fine di descriverne la presenza e il senso, e di far emergere la rilevanza della pratica del dialogo sia sul piano biografico che su quello più strettamente relativo alla scrittura.

\section{Dialogo e vita}

C'è in Michelstaedter, come rileva Franchi, un'«intenzione dialogica della scrittura» (Michelstaedter 2000: 87) sempre legata al piano esistenziale: con questo s'intende che il dialogo non è mai soltanto mera scelta del soggetto che scrive, ma piuttosto una pratica a cui il soggetto che vive accorda l'intero vissuto. Dalle testimonianze biografiche, infatti, Michelstaedter emerge come un assiduo nel dialogo. La sorella Paula Michelstaedter Winteler, nei suoi appunti, ricorda come durante l'ultimo anno del liceo Michelstaedter si fosse avvicinato di più al compagno Enrico Mreule che «aveva già da giovanetto tendenze filosofiche precoci» e lo iniziò «alla ricerca dei valori della vita». A proposito di quel periodo, Paula racconta che «con Mreule e con un altro compagno, Nino Paternolli, il fratello si trovava spesso in una grande soffitta in casa di quest'ultimo, dove passavano delle lunghe sere a discutere problemi seri» (Michelstaedter Winteler 1973: 152); il racconto testimonia di come, già negli anni del liceo, Michelstaedter fosse solito praticare il dialogo con i due compagni Mreule e Paternolli, i futuri protagonisti del Dialogo della salute, coi quali pure spesso saliva sul monte prediletto, il San Valentin.

Il gusto e il desiderio del dialogo non si esauriscono con l'adolescenza, ma rimangono, se si fa eccezione per l'ultimo periodo di silenzio (Michelstaedter Winteler 1973: $162)^{2}$, costanti sempre vive nel modo in cui Michelstaedter conduce l'esistenza. Questi aspetti trovano un riscontro anche nell'abitudine a scrivere lettere, oggi raccolte nell' $E$ pistolario, il «principale testo autografo per la costruzione di un profilo biografico di Carlo Michelstaedter» (Franchi 2014: 15) 3 che non voglia essere solo esteriore ma prestare attenzione anche al «ritmo emotivo del tempo esistenziale» (Franchi 2014: 16).

Nel 1905 Michelstaedter si iscrive alla Facoltà di Matematica dell'Università di Vienna; subito dopo però, ottenuto il permesso dal padre, va a Firenze, dove dopo alcune settimane decide di iscriversi all'Istituto di Studi Superiori. Dal giorno della partenza da Gorizia, che segna il distacco dalla famiglia, tra Michelstaedter e i suoi si instaura subito un rapporto epistolare molto stretto. Lo stesso si verificherà poi con le persone più care, in tutti i momenti di distacco e lontananza. Dice bene Muzzioli quando parla di «investimento di energie nella scrittura epistolare» (Muzzioli 1984: 15), perché dalla partenza da Gorizia alla morte, Michelstaedter scriverà sempre lettere, con maggiore o minore frequenza e a destinatari che variano a seconda dei periodi, ma scrivere lettere

2 La sorella Paula nei suoi appunti biografici racconta di come Michelstaedter negli ultimi mesi fosse poco comunicativo e di come sfuggisse la gente e il contatto con le singole persone intorno a lui.

3 Nella sua ricostruzione biografica Franchi utilizza l'epistolario come fonte primaria, pur non mancando di rilevare che il suo carattere prevalentemente familiare, sommato alle lacune e alle lettere perdute, lo rende un documento molto parziale. 
rimarrà per lui una pratica importante di dialogo con i distanti, uno scambio di voci fatto anche di impeti e attese e tuttavia sentito come necessario. La corrispondenza epistolare con il lontano non è soltanto un mezzo logistico di comunicazione quindi, ma anche, soprattutto negli ultimi anni, il canale tramite cui si esprime profondamente la ricerca del sé e si narrano la propria realtà quotidiana e gli avvenimenti interiori; al tempo stesso, lo scambio epistolare è vissuto con l'impazienza di chi vuol subito arrivare all'altro e l'attesa di chi vuol avere presto dall'altro notizie, perché la costanza del pensiero vorrebbe potersi esprimere sempre e per questo i tempi della corrispondenza postale sono talvolta sentiti come inadeguati: «A chi più si pensa, o si scrive ad ogni istante o non si scrive mai, poiché ogni volta la lettera sembra insufficiente a esprimere questa costanza del pensiero» (Michelstaedter 2010b: 454).

L'Epistolario suggerisce che i dialoghi e le «discussioni filosofiche» non siano stati qualcosa di adolescenziale e confinato nella soffitta di Paternolli. Le lettere a Mreule del 1909-1910, ad esempio, trattano anche tematiche filosofiche che con ogni probabilità i due erano soliti affrontare di persona, almeno fino alla partenza di Mreule; la pratica del dialogo sui valori della vita, tra loro e anche con Paternolli, pare essere una costante negli anni, se in una lettera a Mreule oramai lontano, Michelstaedter afferma: «Di lui [Paternolli] puoi aver gioia: la tua partenza gli è stato l'avvenimento che ha reso incrollabile quanto avevi fondato in lui con le parole e la vita comune. Ora è fatto uomo, ora conosce» (Michelstaedter 2010b: 467). L'impressione che si ha è quella di un sodalizio basato su un dialogo che non mi pare inopportuno chiamare socratico, con Mreule, nel ruolo di Socrate, che era stato capace di render vicine con parola e azione le cose lontane; Michelstaedter gli scrive: «le tue parole si son fatte azione! io mi nutro invece ancora di parole e mi faccio vergogna» (Michelstaedter 2010b: 467, corsivo nel testo). Un rapporto diverso ma per certi aspetti simile, questa volta con Michelstaedter nel ruolo di maestro, è quello con il cugino Emilio, al quale nel settembre 1910 Michelstaedter da Pirano scrive: «Ma ormai ci vedremo presto, ormai sentirò dalla tua voce la parola della fiducia [...]. Potremo parlare a lungo di tante cose e sempre della stessa...» (Michelstaedter 2010b: 472); sono parole che suggeriscono un rapporto profondo fatto di lunghi discorsi che divagavano e al tempo stesso finivano con l'essere ricondotti dai due allo stesso argomento: il come vivere. Nella prefazione alla sua edizione delle Opere, poi, riferendosi alla «relazione intellettuale» dell'inverno 1906-1907 con Nadia Baraden (sulla quale si veda Campailla 2010), Chiavacci afferma che con lei Michelstaedter «sosteneva accanite discussioni filosofiche» (Michelstaedter 1958: VIII).

Questi pochi tratti danno il senso dell'insieme: un'idea dialogica dell'esistenza, tesa tutta alla ricerca e sempre insieme all'altro, grazie all'altro, in uno scambio dialettico sentito evidentemente come necessario, almeno fino all'ultimo periodo, quando Michelstaedter, isolatosi in casa a scrivere la tesi, prese a desiderare di più il silenzio e la solitudine (Michelstaedter Winteler 1973: 162).

Altra esperienza, diversa, del dialogo, è quella che Michelstaedter fa quotidianamente leggendo Platone; dal 1909, infatti, quando era già concordata con il prof. Vitelli la tesi sui concetti di persuasione e retorica in Platone e Aristotele, inizierà a leggere il suo Platone sempre più assiduamente, così come sempre più profonda diverrà la sua familiarità 
coi procedimenti dialettici e la forma dialogica. «Sto bene, batto la campagna, ballo, letto Platone», così in una lettera del febbraio 1909 (Michelstaedter 2010b: 371) e in un'altra del maggio seguente: «mi son messo in questo gran salotto oscuro con la mia piccola lucerna a leggere Platone» (Michelstaedter 2010b: 395). Tuttavia, Platone è «sul tavolo» di Michelstaedter già da molto prima, come si vede da una lettera a Chiavacci del dicembre 1907 (Michelstaedter 2010b: 283). Di Platone discute anche negli scambi epistolari, penso in particolare alle lettere del 1909 a Mreule, che dicono di quanto il dialogo filosofico tra $\mathrm{i}$ due fosse rimasto sempre ben vivo (Michelstaedter 2010b: 379-384; 416-421; 445-449).

Tra le letture non manca nemmeno il Leopardi dei Canti e delle Operette morali. A questo proposito, ricordo che tra le opere a stampa possedute da Michelstaedter ci sono un volumetto dei Canti (Roma, Oreste Garroni, 1905) con postille autografe e un'edizioncina più tarda delle Prose (Roma, Oreste Garroni, 1910); sulle postille rimando al saggio di Campailla Postille leopardiane di Michelstaedter (Campailla 1980: 51-64). Quel Leopardi che Michelstaedter annoverava tra i «"professionisti” del pessimismo» (Michelstaedter 2010b: 417) rappresenta, non solo filosoficamente, uno dei sostrati della scrittura michelstaedteriana.

\section{La scrittura del $t u$}

Prima di trattare della parola e dell'impostazione dialogica della scrittura di Michelstaedter, occorre tornare a riflettere sul valore profondo che egli dà alla vita. Non sono mancati, in sede critica, interventi che hanno tentato di ridimensionare questo valore parlando di nichilismo e solipsismo, stroncando talvolta la parola attraverso la biografia e in particolare interpretando il suicidio come l'atto che decreta la sconfitta del pensiero. Tuttavia, è la parola stessa a recriminare e mostrare l'infondatezza di letture simili. La via della persuasione di cui parla Michelstaedter è sì una via dove ognuno è «il primo e l'ultimo» (Michelstaedter 1995: 36), ma è anche una via dove la pienezza è ricondotta sempre alla relazione con l'altro. Non esiste una persuasione fine a sé stessa, come emerge ad esempio dalle pagine (Michelstaedter 1995: 42-49) con cui si conclude la prima parte de La persuasione e la rettorica, laddove Michelstaedter parla del beneficio e dell'impossibilità di dare senza avere. Cosa significa «dare è avere» (Michelstaedter 1995: 43)? Perché nel Dialogo tra Carlo e Nadia (Michelstaedter 1988a: 95-99) Nadia accusa Carlo di non amarla? Carlo non può amarla perché «non può fare chi non è, non può dare chi non ha, non può beneficare chi non sa il bene» (Michelstaedter 1995: 42). «Chi non ha la persuasione non può comunicarla» (Michelstaedter 1995: 10, corsivo nel testo). Non essere equivale a non poter dare e a non poter amare, perché nell'altro il bisognoso di tutto finisce per amare «solo l'amore di cui ha bisogno» (Michelstaedter 1988a: 98) e dunque non cerca nell'altro che uno "specchio» che «gli dica: "tu sei, io sono, noi siamo"» (Michelstaedter 1995: 58). Questa premessa è imprescindibile: Michelstaedter, parlando della persuasione, non parla di una via individualista, né si tratta di un atteggiamento solipsistico. La sua è una filosofia dell'io in relazione all'altro e al mondo, una parola che perde significato e senso se letta come sterile predicato del sé assoluto, dell'individuo-dio. 
A questa concezione relazionale del singolo, risponde una scrittura impostata dialogicamente in ogni sua forma, l'«intenzione dialogica» che trova espressione non solo nei dialoghi veri e propri, ma anche ne La persuasione e la rettorica, una tesi di laurea vicina per forma al trattato, nelle poesie e negli stessi scambi epistolari. Comunque venga declinata, quella di Michelstaedter è una scrittura del tu e che non esiste quasi senza quel $t u$ continuamente presente nel testo. Il $t u$ chiama in causa l'altro, interroga il lettore, come volesse spingerlo a sondarsi e vedersi; il discorso risulta spesso drammatizzato. Ne La persuasione e la rettorica penso ad esempio all'inizio teso del terzo capitolo, Via alla persuasione, che merita d'esser riportato:

Tí тоŨтo повгіॅ; questo che fai, come che cosa lo fai? - con che mente lo fai? tu ami questa cosa per la correlazione di ciò che ti lascia dopo bisognoso della stessa correlazione, la cui vicinanza non è in te prevista che fino a un limite dato, sicché, a te, schiavo della contingenza di questa correlazione, sia tolto tutto quando a questa cosa questa correlazione sia tolta; e tu debba altra cosa cercare e in balìa della contingenza di questa metterti?

O sai cosa fai? e quello che fai, che è tutto in te nel punto che lo fai, da nessuno ti può esser tolto?

Sei persuaso o no di ciò che fai? Tu hai bisogno che questo avvenga o non avvenga per fare quello che fai, che le correlazioni coincidano sempre, poiché il fine non è mai in ciò che fai, se anche sia vasto e lontano, ma è sempre la tua continuazione. Tu dici che sei persuaso di quello che fai, avvenga che può? - Sì? - Allora io ti dico: domani sarai morto certo: non importa? pensi alla fama? pensi alla famiglia? ma la tua memoria è morta con te, con te è morta la tua famiglia; - pensi ai tuoi ideali? vuoi far testamento? vuoi una lapide? ma domani sono morti, morti anch'essi; - tutti gli uomini muoiono con te - la tua morte è una cometa che non falla; ti rivolgi a dio? - non c'è dio, dio muore con te; il regno dei cieli crolla con te, domani sei morto, morto; domani è finito tutto; il tuo corpo, la tua famiglia, i tuoi amici, la tua patria, quello che fai, quello che ancora puoi fare, il bene, il male, il vero, il falso, le tue idee, la tua parte, iddio e il suo regno, il paradiso, l'inferno, tutto, tutto, domani è finito tutto - fra 24 ore è la morte.

Allora... allora... il dio d'ora non è più quello di prima, non è più quella la patria, quello il bene, quello il male, quelli gli amici, quella la famiglia. - Vuoi mangiare? no, non puoi mangiare, il sapore del cibo non è più quello, il miele è amaro, acido il latte, la carne è nauseante; e poi l'odore, è l'odore che è nauseante: pute di cadavere; - vuoi una donna che ti conforti gli ultimi istanti? no, peggio: è carne morta; - vuoi godere il sole, l'aria, la luce, il cielo? - godere?! - il sole è un'arancia fradicia, la luce è spenta, l'aria irrespirabile, il cielo è una volta bassa che m'opprime... no, tutto è chiuso e buio ormai. - Ma il sole splende, l'aria è pura, tutto è come prima, eppur tu parli come un sepolto vivo che descriva la sua tomba. E la persuasione? non sei persuaso nemmeno della luce del sole, non puoi più muovere un dito, non puoi più tenerti in piedi. Il dio che ti teneva in piedi, che ti faceva chiaro il giorno, e dolce il cibo, che ti dava la famiglia, la patria, il paradiso - quello ti tradisce ora e t'abbandona, poiché è rotto il filo della tua pı৯owuxía (Michelstaedter 1995: 31-32, corsivo nel testo). 
Questo monologo polifonico serrato sembra riprodurre quel «dialogo che l'anima conduce con sé stessa» che è la dianoia (Michelstaedter 1995: 186, corsivo nel testo) ${ }^{4}$ e al tempo stesso chiamare in causa un ipotetico lettore-interlocutore, continuamente apostrofato, interrogato e messo di fronte alle proprie contraddizioni, che sono al tempo stesso le contraddizioni entro cui si dibatte il soggetto autoriale. Il testo procede per domande incalzanti come «questo che fai, come che cosa lo fai? - con che mente lo fai? [...] Sei persuaso o no di ciò che fai?», tramite le quali colui che scrive si rivolge a un tempo a sé stesso e all'altro. Nel suo procedere stringente, la voce dialogante prevede già le risposte, le controbatte e infine mette definitivamente all'angolo l'interlocutore prefigurando una situazione in cui lui che si credeva persuaso, rimanendo senza futuro e dunque senza-dio, finisce per essere un cadavere vivente, fino a quel momento trascinato avanti dal dio del piacere e dalle sue promesse di felicità nel futuro.

$\mathrm{Ne}$ La persuasione e la rettorica questa impostazione dialogica o drammatizzazione del testo è presente e, se talvolta si riduce ad apostrofe o domanda retorica, altrove culmina nel dialogismo. Ci sono casi in cui Michelstaedter immagina le reazioni paurose degli uomini al suo argomentare, le scuse che vigliaccamente si ripetono per continuare a vivere la qualunque vita:

Ma gli uomini dicono: «Questo va bene, ma intanto, intanto bisogna ben vivere»«Intanto»! Intanto che avvenga che cosa? [...] è questione della vita, della vostra vita, della vita d'ognuno [...]» (Michelstaedter 1995: 35, corsivo nel testo).

- «Ma» dicono «io ho le gambe deboli, e quella tua via è impraticabile».

- Ci sono zoppi e diritti - ma l'uomo deve farsi da sé le gambe per camminare - e far cammino dove non c'è strada. [...]

- «Assai abbiamo da portare ognuno la nostra croce perché tu ci venga a imporre l'insopportabile, e a togliere quei sollievi ai quali abbiamo diritto».

- Non portate la croce, ma siete tutti crocefissi al legno della vostra sufficienza [...]» (Michelstaedter 1995: 36-37).

Michelstaedter prefigura l'obiezione degli uomini («dicono») e risponde puntualmente riaffermando il coraggio che occorre a chi vuol mettersi sulla via della persuasione. Pure la pagina conclusiva de La persuasione in qualche modo simula, ma diversamente, un dialogo (Michelstaedter 1995: 131).

Ancor più esemplificativo di questa modalità dialogica di procedere è l'inizio del capitolo La rettorica nella vita, dove viene riportato il dialogo con un «grosso signore». "Vede", mi diceva dopo un pranzo abbondante in conclusione d'un lungo discorso un grosso signore "vede? la vita ha pure i suoi lati belli. Conviene saperla prendere [...]"» (Michelstaedter 1995: 89); di qui il dialogo prosegue senza altri interventi esterni fino alle battute finali e allo "smarrimento» (Michelstaedter 1995: 91) dell'io narrante, che è al tempo stesso uno dei due interlocutori. Al dialogo fa seguito una riflessione dell'autore su questo «uomo del suo tempo», riflessione che nel suo procedere prefigura le reazioni del «grosso signore» e dunque nel suo svolgersi torna a far uso del dialogo:

$4 \quad$ Si tratta dell'espressione con cui Michelstaedter traduce Platone, Sofista, 263 e. 
«Ma» mi direbbe il mio uomo «tutto ciò a me che importa? - io so che sono sicuro e nella coscienza dei miei diritti e dei miei doveri libero e potente». Oppure con le parole di John Stuart Mill (Saggio sulla libertà) «non è qui questione della cosidetta libertà del volere che così inopportunamente viene contrapposta alla dottrina erroneamente detta della necessità filosofica, ma della libertà civile o sociale» (Michelstaedter 1995: 93, corsivo nel testo).

A questa voce immaginata nuovamente fa seguito l'amara riflessione di Michelstaedter: «Della "libertà d'esser schiavo" dunque? E va bene. Infatti è questo che l'uomo cerca, è così che crede giungere alla gioia - né può uscire di sé per vedere di più»» (Michelstaedter 1995: 93). Procedimenti simili, per cui l'autore lega le argomentazioni al dialogismo, o meglio si serve del dialogismo in funzione argomentativa o più genericamente per sostenere una posizione, ritornano anche nelle Appendici critiche, dove Michelstaedter inserisce nel testo «un dialogo che m'è accaduto di sorprendere tra 1" "io" d'un signore [...] e il suo piede» (Michelstaedter 1995: 160); si tratta di un dialogo ispirato a un episodio biografico: «[...] in una gita s'era contuso un piede. [...] Camminava, ma non era completamente guarito dal suo male al piede, per molti mesi ancora ne risentì, e ricordo che a Firenze scrisse uno strano dialogo fra lui e il suo piede ammalato» (Michelstaedter Winteler 1973: 158-159).

L'intenzione dialogica della scrittura non resta confinata all'opera maggiore, ma trova espressione anche nelle poesie e, più di tutto, nei dialoghi. Nelle poesie, non solo torna spesso il $t u$, quasi segno distintivo, ma vengono anche nuovamente inserite parti dialogiche. Penso in particolare a I figli del mare (Michelstaedter 1987: 79-84), dove la narrazione viene interrotta dal dialogo tra Itti e Senia (sbilanciato in realtà a favore di Itti, l'interlocutore forte) e dal richiamo del «domestico focolare» (Michelstaedter 1987: 82-83). Anche le liriche A Senia (Michelstaedter 1987: 85-96) ruotano tutte intorno al $t u$, che corrisponde questa volta alla dedicataria Argia-Senia, a cui l'io poetante si rivolge. A completare il quadro restano gli scritti in forma dialogica, come Il dialogo della salute, il dialogo tra Carlo e Nadia, il dialogo tra Diogene e Napoleone, il dialogo tra la cometa e la Terra e altri dialoghetti minori: qui il dialogo non è inserito a contaminare un altro genere letterario, ma scelto come forma di scrittura.

\section{Il dialogo come forma della dialettica}

La domanda con cui vorrebbe idealmente concludersi questo contributo è: perché il dialogo? Qual è il senso della scrittura dialogica praticata da Michelstaedter? Come emerge dagli aspetti analizzati nelle sezioni precedenti, non è possibile interpretare la pratica dialogica nella scrittura in senso univoco, perché molti sono gli elementi da tenere in considerazione; tuttavia, si tratta di una forma che deriva in primis da un pensiero filosofico-esistenziale concreto e desideroso di farsi pratica in ogni declinazione possibile, anche in quella della scrittura.

5 Michelstaedter parla più volte dell'episodio e del piede nelle lettere, dall'aprile all'ottobre del 1909 (Michelstaedter 2010b: 381, 385, 399, 401, 408-409, 412, 416, 436), si trattò dunque di un problema che lo afflisse e limitò per molto tempo. 
Le riflessioni sul vissuto biografico hanno fatto emergere la comunicazione ininterrotta tra vita e opera; questo scambio continuo, se da un lato impedisce di immaginare scrittura e vita come mondi lontani, dall'altro evidenzia la forte connessione tra il dialogo socratico - ovvero rivolto alla ricerca della verità - praticato con l'altro e il dialogo praticato nelle dimensioni della scrittura. Se la relazione con l'altro per essere vera ha bisogno di configurarsi come relazione dialettica basata sul dialogo, al tempo stesso gli scritti, nella loro forma più coerente - come argomenterò meglio di seguito a proposito del Dialogo della salute - sono dialoghi o testi contaminati dal dialogismo.

Non meno importante, per comprendere la genesi e il senso dei dialoghi, è l'influenza di alcune delle letture fondamentali per Michelstaedter; la scelta dialogica pare strettamente legata, in primo luogo, ai modi di autori frequentati e meditati a lungo come Platone e Leopardi. I dialoghi platonici e le Operette morali non vanno tuttavia considerati soltanto come modelli letterari, sia perché l'attrattiva della forma viene per Michelstaedter dall'attrattiva del pensiero, sia perché sono veicoli non tanto e non solo di un genere quanto di una strategia discorsiva che rinnova il linguaggio filosofico, salvandolo dai rischi della retorica: il linguaggio e la sua forma devono restare quelli del pensiero che si svolge tra gli uomini che cercano, non porsi come il mezzo con cui chi sa - come Aristotele - comunica verità assodate sugli uomini e sul mondo.

Di qui si vede come le ragioni più profonde della scelta dialogica siano filosofiche molto più che legate a un'assimilazione formale: la personalissima percezione delle potenzialità del dialogo come genere della ricerca, accompagnata al rifiuto dei generi precostituiti di scrittura, porta Michelstaedter a praticare una modalità affatto usuale ma evidentemente sentita vicina e adatta a comunicare un sapere mai dato e sempre ricercato dialetticamente. Al tempo stesso, scrivere dialoghi è per Michelstaedter una risposta al desiderio forte di tornare a Socrate e alla dialettica - unica forma di educazione possibile.

Questo senso profondo della forma dialogica può essere chiarito richiamando l'inizio della prefazione a La persuasione e la rettorica, un breve testo che l'autore infine escluse dalla tesi di laurea ma di cui non si può non tenere conto.

«Io lo so che parlo perché parlo ma che non persuaderò nessuno; e questa è disone-

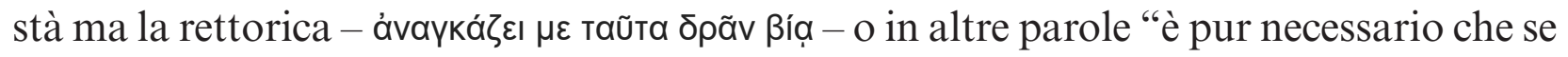
uno ha addentato una perfida sorba la risputi"» (Michelstaedter 1995: 3). Queste poche righe chiariscono la tragicità cui si lega uno scritto come La persuasione e la rettorica: Michelstaedter sa che scrivendo non persuaderà nessuno e dunque il suo atto - scrivere nonostante questa consapevolezza - si configura come disonesto. Tuttavia, troppo grande è l'urgenza di dire quello che pure molti altri prima di lui avevano detto senza riuscire mai a cambiar niente: chi ha addentato l'amara verità, non può far a meno di risputarla, come il prigioniero non può far a meno di tornare nella caverna e provare a salvare. La persuasione e la rettorica è una tesi di laurea scritta in una forma che, sebbene non classificabile, si avvicina a quella del trattato; a questa forma di scrittura, si contrappongono Il dialogo della salute e gli altri dialoghetti.

Questa osservazione dà modo di confermare le ipotesi interpretative già avanzate in merito alla tendenza di Michelstaedter al dialogismo, anche laddove avrebbe po- 
tuto replicare, ad esempio, i modi più trattatistici dell'opera maggiore. Nel caso del Dialogo della salute, in particolare, la scelta della forma dialogica risulta chiaramente correlata alle finalità del testo. La persuasione e la rettorica è uno scritto rivolto, nelle intenzioni prime, alla commissione di professori di fronte alla quale Michelstaedter avrebbe dovuto discutere la tesi di laurea. Il dialogo della salute si presenta invece come «l'altro lato dell'iperbole», rispetto all'opera maggiore: non uno scritto legato a un dovere, ma uno scritto spontaneo e libero da costrizioni di ogni tipo; non un'opera indirizzata a uomini che incarnavano la tanto odiata retorica, ma un'opera dedicata al cugino Emilio e a quanti giovani, come lui, non avessero ancora messo il loro dio nella loro carriera: a quanti come lui potessero ancora essere salvati, in quanto esseri non ancora "incamiciati", anime in fieri che non avevano insomma subito la cristallizzazione sociale e ormai ridotto la propria vita a forma fissa. Il dialogo viene qui sentito come più adatto allo scopo: l'educazione ${ }^{6}$ del cugino Emilio, emblema di tanti giovani - ovvero un fine paideudico. Nelle Appendici critiche Michelstaedter scrive che «l'educazione socratica [...] è creatrice d'uomini» e che è «l'unica» che «dà all'uomo le gambe per camminare» (Michelstaedter 1995: 150, corsivo nel testo). In questo senso, il dialogo si profila come una forma di scrittura più coerente e meno disonesta rispetto a quella de La persuasione e la rettorica, nonché come la forma meno violenta e più vicina alla dialettica socratica, al vivo dialogare del filosofo, che solo poteva salvare.

Se la storia, fin dall'ultimo Platone, è vista da Michelstaedter come un tradimento della pura ricerca socratica, basata sul $\tau$ í $̇ ં \tau \imath$, e un'affermazione violenta della «rettorica scientifica» e del «sapere costituito» che hanno preso il posto dell'originaria domanda di verità, all'opposto il dialogo si configura come una via per tornare a Socrate; Il dialogo della salute, in questo senso, è il modo in cui Michelstaedter tenta la fedeltà al sapere-non-costituito e alla dialettica socratica, da cui soltanto crede possa venire la verità?.

\section{BIBLIOGRAFIA}

CAMPAILLA, Sergio (1980): Scrittori giuliani. Bologna: Pàtron.

(1988): Della salute ovvero della malattia, in Michelstaedter 1988a: 9-21.

(2010): Il segreto di Nadia B. La musa di Michelstaedter tra scandalo e tragedia. Venezia: Marsilio.

\footnotetext{
6 Va intesa in senso socratico. Michelstaedter distingue chiaramente l'educazione (la modalità socratica, appunto) dall'ammaestramento (la modalità dell'ultimo Platone e di Aristotele), altresì detto «educazione civile»: mentre l'educazione «sommuove dall'intimo l'individuo»e gli impone «l'identità con sé stesso (persuasione)», l'ammaestramento «vuol foggiare da ogni uomo un dato istrumento che giovi per dati usi, non perché basti a sé stesso» (Michelstaedter 1995: 148) ovvero vuol fare dell'uomo «un degno braccio irresponsabile della società (Michelstaedter 1995: 130)».

7 Per quanto riguarda la debolezza dialettica del Dialogo della salute, su cui non mi sono soffermata poiché più che gli esiti interessavano in questa sede le motivazioni della scelta dialogica, rimando a Tanzi 2003: 143147, che parla di «monologo polifonico»; Campailla 1988: 11-18, che vede il Dialogo come la «drammatizzazione di un monologo»; Micheletti 2003: 77-86.
} 
CERRUTI, Marco (1987): Carlo Michelstaedter. Milano: Mursia.

FRANCHI, Gian Andrea (2014): Una disperata speranza. Profilo biografico di Michelstaedter. Milano - Udine: Mimesis.

MEROI, Fabrizio (2011): Persuasione ed esistenza. Filosofia e vita in Carlo Michelstaedter. Roma: Edizioni di Storia e Letteratura.

MICHELETTI, David (2003): "Dianoia, ovvero il dialogo che l'anima conduce con se stessa. Su Il dialogo della salute di Carlo Michelstaedter". Davar, n. 1: 77-86.

MICHELSTAEDTER, Carlo (1912): Dialogo della salute. "Poesie", a c. di V. Arangio-Ruiz. Genova: Formiggini.

(1958): Opere, a c. di G. Chiavacci. Firenze: Sansoni.

(1987): Poesie, a c. di S. Campailla. Milano: Adelphi.

(1988a): Il dialogo della salute e altri dialoghi, a c. di S. Campailla. Milano: Adelphi.

(1988b): Il dialogo della salute, a c. di G.A. Franchi. Bologna: Agalev.

(1995): La persuasione e la rettorica. Appendici critiche, a c. di S. Campailla. Milano: Adelphi.

(2000): Il prediletto punto d'appoggio della dialettica socratica e altri scritti, a c. di G.A. Franchi. Milano: Mimesis.

(2010a): Dialogo della salute e altri scritti sul senso dell'esistenza, a c. di G. Brianese. Milano - Udine: Mimesis.

(2010b): Epistolario, a c. di S. Campailla. Milano: Adelphi.

MICHELSTAEDTER WINTELER, Paula (1973): Appunti per una biografia di Carlo Michelstaedter, in appendice a Campailla, Sergio: Pensiero e poesia di Carlo Michelstaedter. Bologna: Pàtron, 147-164.

MUZZIOLI, Francesco (1984): “Il vociano Michelstaedter". Alfabeta, n. 57: 15.

TANZI, Roberta (2003): "Dialogo e dialogicità in Michelstaedter: per una lettura del Dialogo della salute (e una controlettura de La persuasione e la rettorica)»". Studi goriziani, voll. XCVII - XCVIII: 137-150.

\section{PROFILO ACCADEMICO E PROFESSIONALE}

Claudia Zavaglini (Fermo, 1/6/1987) è dottoranda in Letterature romanze (Italianistica) presso il Dipartimento di Lingue e Letterature romanze dell'Università Palacký di Olomouc (Repubblica ceca), sotto la supervisione del prof. Jiř́ Špička. Sta svolgendo il dottorato in cotutela con l'Università di Perugia (Italia), sotto la supervisione del prof. Sandro Gentili.

La sua tesi di dottorato ha per tema il dialogo come forma di scrittura nella letteratura moderna e in particolare nel Novecento italiano; la sua ricerca mira dunque a descrivere la presenza e le declinazioni del genere dialogico nel XX secolo, soffermandosi sugli esempi più degni di attenzione: Michelstaedter, Silone, Pavese, Moravia e altri. Da un punto di vista teorico, il suo lavoro intende descrivere il genere dialogico nelle sue caratteristiche e peculiarità, al fine di proporre una teoria e poetica del dialogo italiano 
moderno.

Dal 2014 al 2017 ha tenuto corsi di lingua e letteratura italiana presso l'Università di Olomouc.

Ha partecipato a conferenze internazionali con comunicazioni su Carlo Michelstaedter, al quale ha lungamente dedicato i suoi studi, e sul dialogo letterario. Attualmente si sta occupando dei Dialoghi con Leucò di Cesare Pavese.

Fecha de recepción del artículo: 19-12-2017

Fecha de aceptación del artículo: 12-05-2018 